\title{
In situ Metabolite Mass Spectrometry Imaging: New Insights into the Adrenal Gland
}

Authors

Fengxia Li, Annette Feuchtinger, Axel Walch, Na Sun

\begin{abstract}
Affiliation
Research Unit Analytical Pathology, German Research Center for Environmental Health, Helmholtz Zentrum München, München, Germany
\end{abstract}

Key words:

mass spectrometry imaging, adrenal gland, steroid

hormones, catecholamines, metabolite imaging

$\begin{array}{lr}\text { received } & 16.12 .2019 \\ \text { accepted } & 18.02 .2020\end{array}$

Bibliography

DOI https://doi.org/10.1055/a-1129-6947

Published online: 29.4 .2020

Horm Metab Res 2020; 52: 435-447

(c) Georg Thieme Verlag KG Stuttgart · New York

ISSN 0018-5043

Correspondence

Na Sun

Research Unit Analytical Pathology, German Research Center for Environmental Health, Helmholtz Zentrum München Ingolstaedter Landstrasse 1

85764 Neuherberg

Germany

Tel.: + 49893187 4133, Fax: + 498931873349

na.sun@helmholtz-muenchen.de

\section{ABSTRACT}

The adrenal gland integrates catecholamine-producing neuroendocrine cells and steroid-producing cells with mesenchymal origin in a structured manner under one capsule and is a key regulator for vital bioactivity. In addition to adrenal-specific disease, dysregulation of adrenal hormones is associated with systemic effects, leading to undesirable metabolic and cardiovascular consequences. Mass spectrometry imaging (MSI) technique can simultaneously measure a broad range of biomolecules, including metabolites and hormones, which has enabled the study of tissue metabolic and hormone alterations in adrenal and adrenal-related diseases. Furthermore, this technique coupled with labeled immunohistochemistry staining has enabled the study of the pathophysiological adaptation of the adrenal gland under normal and abnormal conditions at different molecular levels. This review discusses the recent applications of in situ MSI in the adrenal gland. For example, the combination of formalin-fixed paraffin-embedded tissue microarray and MSI to tissues from patient cohorts has facilitated the discovery of clinically relevant prognostic biomolecules and generated promising hypotheses for new sights into physiology and pathophysiology of adrenal gland. MSI also has enabled the discovery of clinically significant tissue molecular (i. e., biomarker) and pathway changes in adrenal disease, particularly in adrenal tumors. In addition, MSI has advanced the ability to optimally identify and detect adrenal gland specific molecules. Thus, as a novel analytical methodology, MSI has provided unprecedented capabilities for in situ tissue study.

\section{Introduction}

Matrix-assisted laser desorption/ionization mass spectrometry imaging (MALDI-MSI) is a technique that can directly identify and localize hundreds to thousands of molecules in a tissue sample simultaneously, including metabolites, lipids, glycans, peptides, proteins, glycolipids, and drugs and their metabolites [1]. The molecular distribution can then be correlated with the histomorphology. Unlike conventional molecular analysis methods of tissue samples [e.g., immunohistochemical (IHC) and histochemical techniques], which specifically detect individual compound localizations via antibody applications or compound classes such as polysaccharides [2], MSI-based techniques provide a simultaneous label-free detection of various types of species in the same tissue section. The cell-specific molecular signatures accessible through
MSI are hugely complementary to histopathological, IHC and other established molecular (e. g., genomics-related) data [1,3] and therefore offer a major advantage for synergizing molecular information and morphology. Consequently, MSI has rapidly and substantially impacted basic and clinical research by identifying molecular changes associated with disease. MSI is a promising tool to expand the field of molecular pathology to improve diagnostics, prognostics, and theranostics for various tumor types [4-6].

Metabolomics can be broadly defined as the comprehensive analysis of all small molecule metabolites (i. e., $\leq 1500 \mathrm{Da}$ ) in a biological system. These small molecular weight compounds, including small peptides, oligonucleotides, sugars, nucleosides, organic acids, ketones, aldehydes, amines, amino acids, lipids and steroids, are present in cells, tissues and/or organisms [2]. Metabolism al- 
teration occurs in various human diseases, and metabolic reprogramming is a core hallmark of cancer [7-9]. Qualitative and quantitative analysis of metabolites in biological systems are facilitated by various analytical methods, particularly MS (e. g., MALDI-MS, liquid chromatography-MS and gas chromatography-MS) [9]. Among these and other frequently used analytical assays, which usually homogenize and extract the species, MSI is the leading technique for conducting spatially resolved metabolome measurement either in fresh frozen (FF) tissue sections or formalin-fixed paraffin-embedded (FFPE) tissue $[10,11]$. The preparation of FF samples can be particularly difficult (i. e., collection, handling and storage in the routine setting), whereas the vast majority of FFPE specimens (biopsies and surgical resections) are prepared in pathology laboratories. Several investigators have conducted MALDI-MSI analysis of FFPE tissue specimens (reviewed in $[2,10]$ ). A proof-of-principal study of the spatial and chemical conservation of metabolites in archived FFPE tissue samples reports that $72 \%$ of the $m / z$ species overlap between FFPE and FF tissue sections [11]. Based on this work, a detailed analytical protocol for measuring molecular spatial distributions in FFPE samples using MALDI Fourier-transform ion cyclotron resonance (FTICR) MSI platform has been published [12]. The validity of the MSI protocol for FFPE tissues was demonstrated by a multicenter interlaboratory round robin study which showed a high level of between-center reproducibility of FFPE tissue metabolite data [13].

The adrenal gland plays a key role in the regulation of metabolic, cardiovascular, immune, neuronal, and mental processes through the action of two major types of hormones, steroids and catecholamines, which are produced in the outer cortex and inner medulla regions, respectively. Although both tissues of the adrenal gland descend from different origins during embryonic development and produce different types of hormones, the adrenal cortex and medulla interact with and influence each other under both healthy and pathological conditions in multiple ways, including through the blood flow that delivers steroids collected in the cortex region to the medulla and through contact between different cells at the cortical-medullary junction region [14]. In addition, the adrenal gland is in bidirectional crosstalk with the major systemic functions in the context of systemic disease, such as obesity and inflammation. Metabolites can interact directly or indirectly with molecular targets, influencing the risk and complications associated with adrenal gland disease or with adrenal dysfunction. The functional integrity of this organ system therefore is essential for hormonal homeostasis, and adrenal dysfunction may contribute to various metabolic and cardiovascular disorders and stress-related diseases [15-18].

As an emerging tool and leading technique for spatially resolved molecular assessment, MSI facilitates the study of the critical role of the adrenal gland in physiological and pathophysiological conditions. This technology fills the gap for visualizing the distributions of hormones, metabolites and drugs in the cortex and medulla of the adrenal gland and other organs or tissues from animal models to patients. Mapping metabolites and hormones to their respective pathways provides a means to image the activities of pathways in tissues [19-21]. Molecular and metabolomic pathway changes associated with adrenal diseases can be discovered under the context of their highly structured histology and interactive functionality among diverse cortical and medullary cell components by this methodology. Importantly, the tissue section remains intact during MALDI analysis, which allows for further co-registration analyses to be conducted after the measurement has been taken. This feature therefore provides great potential for addressing highly interesting and unanswered questions in the study of the adrenal gland. The precise localization of species within specific cells may enable the study of the aforementioned spatial and functional interaction between the cortex and medulla regions. In this review, we described the recent applications of in situ mass spectrometry imaging in adrenal gland study, including molecular imaging of adrenal gland physiology as well as pathophysiological molecular changes of adrenal diseases. MSI provides an advanced methodology for gaining a deeper insight into adrenal function, as well as clinically relevant tissue molecular and functional adaptation in adrenal-related disease.

\section{In situ MALDI-MSI used in tissue studies}

MSI was developed from secondary ion mass spectrometry (SIMS), which introduces a pulsed ion beam moving across the sample's surface to generate a secondary ion to be directed to the mass spectrometer inlet $[22,23]$. Among various sample introduction methods, matrix-assisted laser desorption/ionization (MALDI) is one of the most commonly applied ionization techniques coupled with MSI analysis, and was applied to biological tissue sections in the mid-to-late 1990s. MALDI-MSI was first used to visualize proteins and then quickly was extended to other endogenous and exogenous molecular classes by utilizing other matrices and sample preparation optimization [23-26]. MALDI-MSI has been a powerful tool for metabolome study in tissue samples. In a typical MALDI-MSI approach, tissue sections are mounted on a MALDI-compatible target carrier and then coated with a matrix [27]. The laser beam probes a specific location on the matrix-coated tissue in a predefined raster. The matrix absorbs and transfers laser energy to the analytes, thus enabling desorption and ionization. A mass spectrum is therefore generated at each measurement spot. The generated MS data can then be co-registered to the histologically stained section to enable visualization of the molecular content within a tissue morphological context [12]. Depending on the molecular classes of interest, pre-treatment of samples may be necessary to detect specific compound/compound classes or to improve sensitivity. These pre-treatments include washing to attenuate the influence of high abundance compounds on low abundant molecules, on-tissue digestion for protein or glycan analysis, and on-tissue derivatization to improve sensitivity for specific drugs or metabolites [28-30].

Post-measurement data processing and statistical analysis are a key step in MALDI-MSI analysis [23, 31]. Processing of MALDI-MSI data must consider the nature of spatially resolved mass spectra for each sample, with each mass spectrum containing hundreds to thousands of mass signals [31,32]. The basic data processing pipeline typically involves raw data exporting, pick alignment, pick peaking, and peak annotation. Peak annotation can be done either by MS/MS experiments directly from tissue and/or by a database search of measured $m / z$ values [23]. The commonly used public databases for MSI annotation of small molecules are HMDB (http:// www.hmdb.ca/), METLIN (https://metlin.scripps.edu), lipid maps (http://www.lipidmaps.org/), and/or METASPACE (http://annot ate. 
metaspace2020.eu) [33, 34], Accurate mass measurement, coupled with tandem MS, enables small endogenous compounds to be unambiguously identified directly from the tissue [28].

Identification of metabolites is a continually developing field in metabolomics. Comparison of the mass spectral data against literature and databases is important for metabolite annotation. The continual development of metabolite databases, bioinformatics tools and MS instrumentation lead to improvements in metabolite annotations and structural identifications [35]. It is a challenge to identify and characterize the structures of unknown metabolites that are not available in databases. In silico analyses have been developed to assist with identification of metabolites for which MS data are not available in databases. In these cases, analysis of the fine isotope pattern of the $\mathrm{m} / \mathrm{z}$ of interest using Bruker DataAnalysis and SmartFormula can be useful to predict molecular formula and possible unknown metabolite structures [11,12]. Furthermore, it may be necessary to identify unknown metabolites using nuclear magnetic resonance (NMR) analysis or extensive chemical synthesis to enable structural comparisons using tandem mass spectrometry (MS/MS) [36, 37].

Due to the amount of MSI data (i. e., in the gigabyte range), the processing and handling of the data have become computationally demanding. Commercial software packages, such as Multimaging (Imabiotech, France) or SCiLS (Bruker Daltonics, Germany), can handle terabyte-sized, multi-sample data sets and include many statistical tools for biomarker discovery [23]. In addition to classical statistical tests, supervised and/or unsupervised statistical analyses have been applied to MSI data analysis [31, 38-42]. A schematic of the MALDI-MSI concept with post-measurement data handling is provided in > Fig. 1, [43].

The application of MSI to research questions of biological and clinical significance has increased exponentially since the introduction of the technology [28]. MSI has been applied by many studies to investigate complex structures in tissue, including in brains, eyes, joints, wounds and atherosclerotic plaques. Oncology, however, has been and still is the driving field for MSI. In this context, MSI has been used to extract tumor-specific molecular information for the purpose of biomarker discovery to improve diagnosis and prognosis as well as to study intra-tumor heterogeneity and classification of tumor margins. Its applications generally cover all stages of translational research from pre-clinical to clinical studies [46, 23, 44-47].

MSI analysis has enabled access to a variety of molecules in the adrenal gland. In the healthy human adrenal gland, a wide spectrum of endogenous metabolites, including catecholamines, sterol and steroid metabolites, nucleotide derivatives, intermediates of glycolysis and the tricarboxylic acid (TCA) cycle and lipids and fatty acids, have been measured by MALDI-FTICR-MSI analysis [20]. Under pathophysiological conditions, endogenous metabolites and various hormones and their derivatives (e. g., 18-hybrid steroids) also were detected in FFPE specimen from adrenal tumors [21, 48, 49]. These findings have led to deeper insight into the metabolites and hormone adaptation in the functional and dysfunctional adrenal gland and adrenal tumors as will be shown in later cases. Specific to the adrenal gland, a few studies also have focused on the improvement of the detection and localization of adrenal-specific hormones, including for catecholamines [50-52] and particularly for poorly ionized and isomeric steroids, leading to new hypothesis of adrenal disease pathogenesis, as will be reported later in this review $[49,52,53]$.

\section{MSI method adaption for visualizing steroids in adrenal cortex (FF tissue)}

Although high-resolution mass analyzers such as FTICR-MS (i. e., 100000 mass resolution for common tissue metabolites) allow relatively accurate and confident assignments of ion peaks to chem-

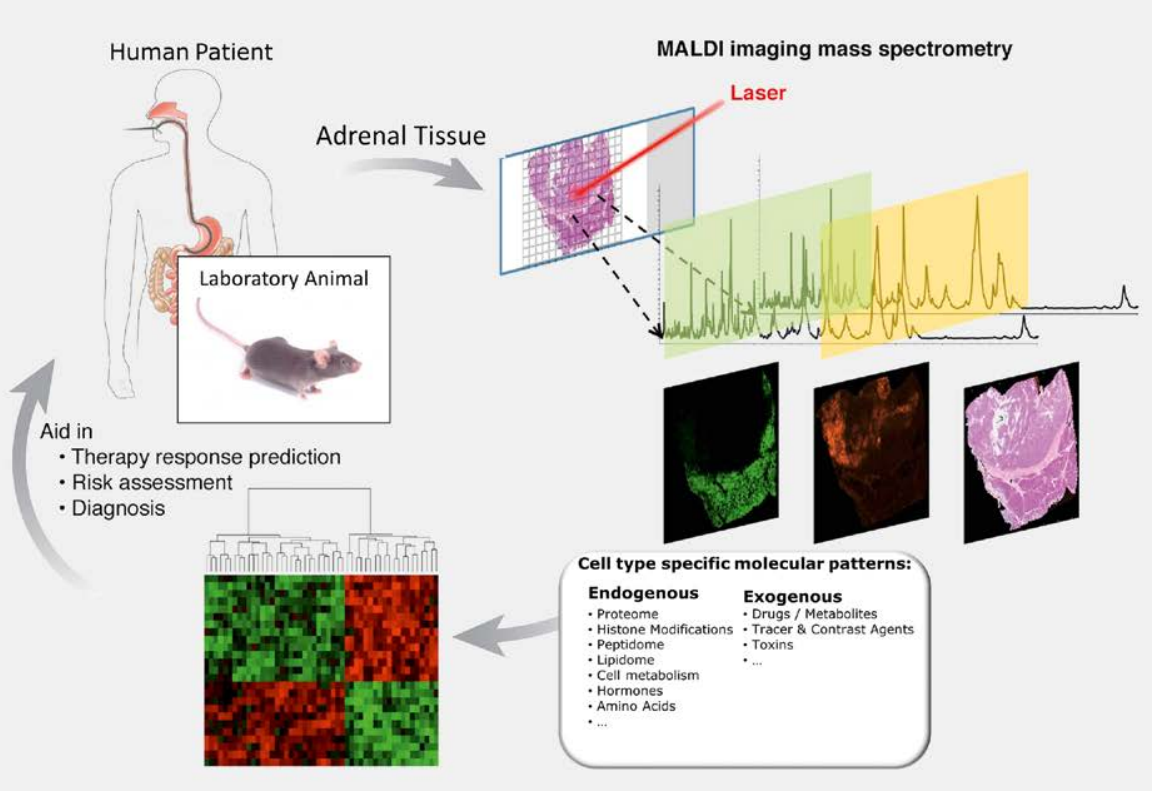

- Fig. 1 Representation of the matrix assisted laser desorption/ionization mass spectrometry imaging (MALDI-MSI) workflow: matrix deposition, in situ laser desorption mass spectrometry analysis, hematoxylin and eosin $(\mathrm{H} \& \mathrm{E})$ staining, and co-registration of MSI data with the $\mathrm{H}$ \& $\mathrm{E}$ image to achieve $\mathrm{H} \&$ E-guided extraction of mass spectra information. (Reprinted from [43] with permission from Elsevier) [rerif]. 

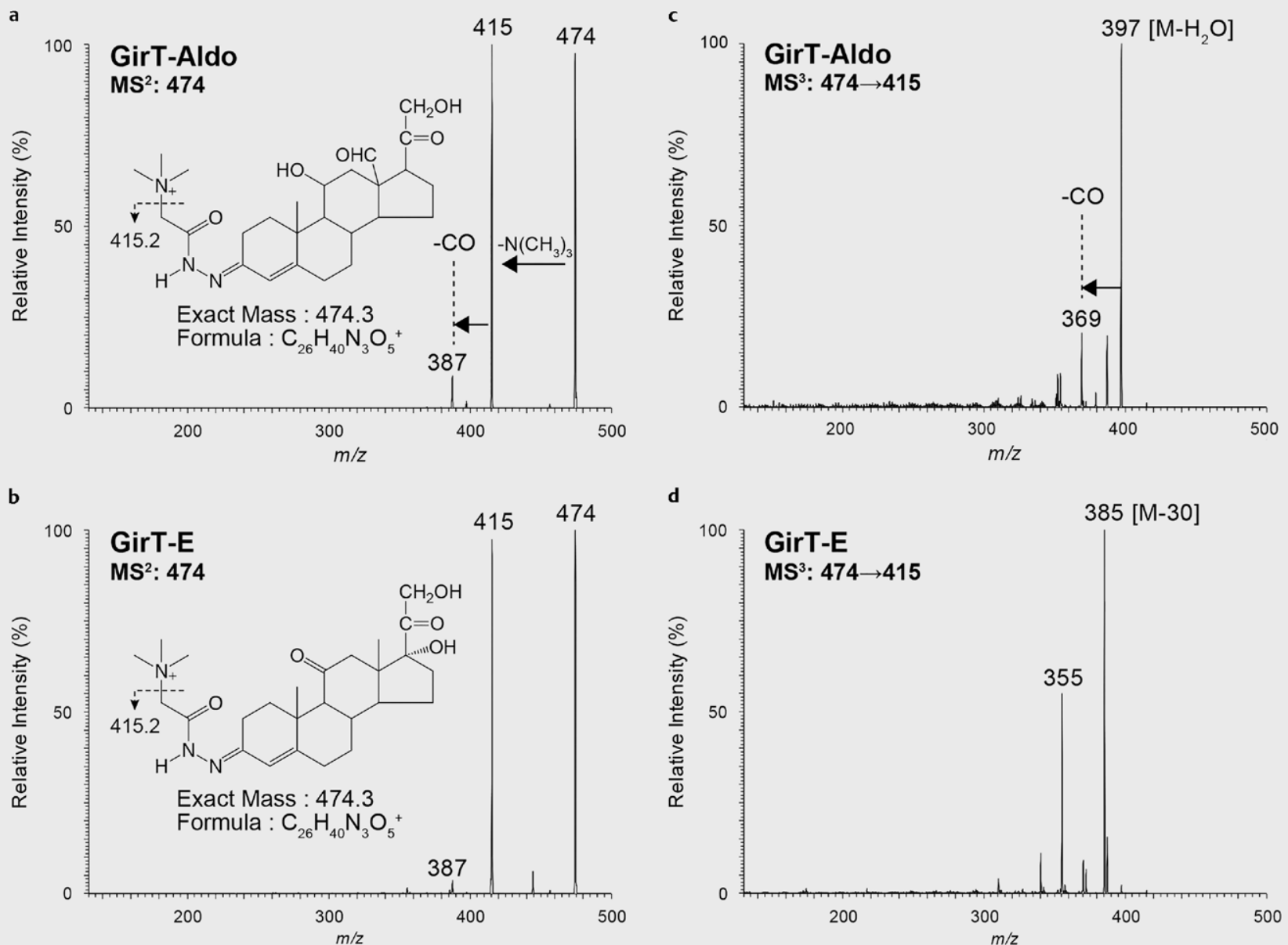

- Fig. 2 Product ion spectra of GirT-aldosterone and GirT-cortisone (GirT-E) standards. MS² spectra of $\mathrm{m} / \mathrm{z} 474$ and presumed structures show similar fragmentation patterns for a GirT-aldosterone and $\mathbf{b}$ GirT-cortisone. $\mathrm{MS}^{3}$ spectra of $\mathrm{m} / \mathrm{z} 415$ show characteristic fragmentation patterns for $\mathbf{c}$ GirT-aldosterone and d GirT-cortisone. (Reprinted from [52] with permission from American Chemical Society) [rerif].

ical formulas, a lack of detection sensitivity can hinder its application to the molecular imaging due to low ionization efficiency, ion suppression by abundant species, and background spectral interference from either the matrix and/or tissue components [51, 52]. Low sensitivity in the adrenal gland MSI study is particularly prominent for neutral steroids as they have relatively low abundance, poor ionization yields (i. e., lack of hydrogen donor or acceptor moieties), and susceptibility to ion suppression of lipids and proteins [53]. Another challenge for MSI molecular assignment is that it cannot discriminate between structural isomers, which occur frequently for steroids [52]. Four pairs of structural isomers can be found in human adrenocortical steroidogenesis pathways, including 18-hydroxyl corticosterone and cortisol, cortisone and aldosterone, corticosterone and 11-deoxycortisol, and 11-deoxycorticosterone and 17-hydroxyprogesterone. Differentiating these steroids during in situ on-tissue MSI analysis is challenging but provides the opportunity to study their biological activity and communication within their producing context. With regard to these two major challenges, in situ chemical derivatization in combination with tandem MS techniques has demonstrated its usefulness in steroid imaging in the adrenal gland. Chemical derivatization introduces polar functional groups resulting in enhanced ionization yields, whereas tandem mass fragmentation produces characteristic fragments that are particularly useful for structure identification and differentiation of structural isomers.

To enhance the sensitivity of neutral steroid MSI analysis, Cobice et al. explored derivatization methods and found that on-tissue chemical derivatization using GirT reagent has the highest sensitivity for 11-dehydrocorticosterone and corticosterone, achieving low limit of detection and high signal-to-noise ratio ( $>100)$ [53]. Such derivatization reactions target the $\alpha, \beta$-unsaturated ketone at $\mathrm{C} 3$ in the steroid $\mathrm{A}$-ring. Cobice et al. used collision induced dissociation (CID)/liquid extraction surface analysis to confirm the structure of the generated compounds. CID of the GirT derivatives produced a series of fragment ions characterized by the loss of the quaternary amine tag $[\mathrm{M}-59]^{+}$and carbon monoxide $[\mathrm{M}-87]^{+}$ of the derivatized group. This method was applied to image 11-dehydrocorticosterone and corticosterone distribution in the mouse adrenal gland and brain. MSI analysis of the mouse brain with glucocorticoid-amplifying enzyme 11ß-HSD1 deficiency or inhibition 

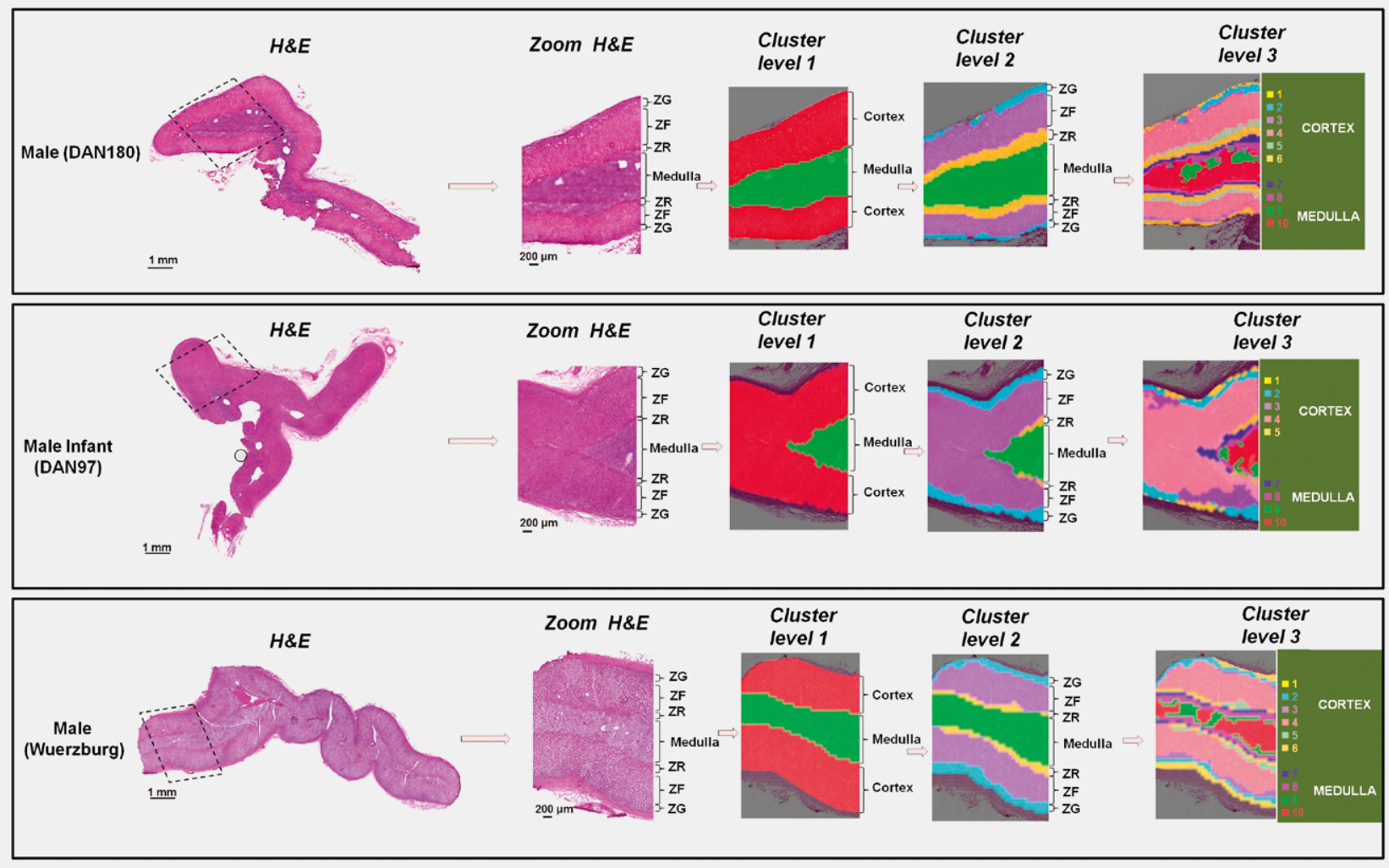

- Fig. 3 Unsupervised hierarchical clustering reveals the molecular structures of the adrenal gland. These structures match well the histology of the adrenal gland in level 1 (two classes separate the cortex and medulla) and level 2 [four classes separate the zona glomerulosa (ZG), zona fasciculata (ZF), zona reticularis (ZR), and medulla], and identified additional distinct molecular layers in level 3 (10 molecular classes). Scale bars: 1 mm. ZG: Zona glomerulosa; ZF: Zona fasciculate; ZR: Zona reticularis; H \& E: Hematoxylin and eosin. (Reprinted from [20] by permission of Oxford University Press) [rerif].

revealed sub-regional corticosterone/11-dehydrocorticosterone ratio changes, and accumulation of 7-ketocholesterol as the alternative $11 \beta$-HSD1 substrate. This MSI data was validated by liquid chromatography (LC)-MS/MS in whole brain homogenates with high correlation.

Recently, GirT derivatization followed by tandem MSI (MS/MS: $\mathrm{MS}^{2}$ and MS/MS/MS: $\mathrm{MS}^{3}$ ) successfully differentiated aldosterone from its isomer cortisone, which shows a different distribution pattern. $\mathrm{MS}^{3}$ was necessary for generating characteristic fragments to distinguish aldosterone from cortisone, as shown in $>$ Fig. 2, [52]. As the first studies to visualize the localization of aldosterone on a frozen rat adrenal section, MSI analysis confirmed its production in the ZG layer following a sodium-deficient diet [49]. GirT derivatization has also enabled the visualization of other steroids of GirT-corticosterone, GirT-18-hydroxycorticosterone and GirT-progesterone [49]. This method has been applied to the study of aldosterone-producing cell clusters (APCCs), which occur in more than $80 \%$ of adult adrenals ( $>30$ years old), and their relation to APAs as will be discussed later $[49,54]$. Subsequently, this derivatization and $\mathrm{MS}^{3}$-based method was extended to separate not only aldosterone but also cortisol from their isomers, cortisone and 18 -oxocorticosterone, respectively. Cortisol distribution was visualized for the first time in the human adrenal gland [52].
For the detection of steroids, publications are mainly focused on chemical derivatization using FF tissue samples which could increase ionization efficiency of steroids. Nevertheless, previous studies have demonstrated that certain steroids can be detected in FFPE tissue without specific chemical derivatization $[21,48]$.

\section{Molecular imaging of adrenal gland physiology by MSI (FF tissue)}

The normal functional anatomy of the adrenal gland has been studied using MSI techniques to look into their metabolites and hormone profile in situ. Localization of cholesterol and corticosterone with a sub-cellular spatial resolution was demonstrated in the rat adrenal cortex using imaging time-of-flight secondary ion mass spectrometry (TOF-SIMS) [55]. Wu et al. applied desorption electrospray ionization (DESI) imaging (positive ion mode, raster size $200 \mu \mathrm{m}$ ) to porcine and rabbit adrenal gland tissue and determined that the distribution of the small hormones epinephrine and norepinephrine enriched in the medulla layer [56]. Using MALDI-FTICR-MSI in both the positive and negative ion modes (raster size $200 \mu \mathrm{m}$ ), Wang et al. determined the spatial distribution pattern of 544 lipids and 11 non-lipid metabolites in porcine adrenal glands, and showed that many classes of lipids had distinct distribution patterns in different functional zones of the adrenal gland [57]. Another study has in- 


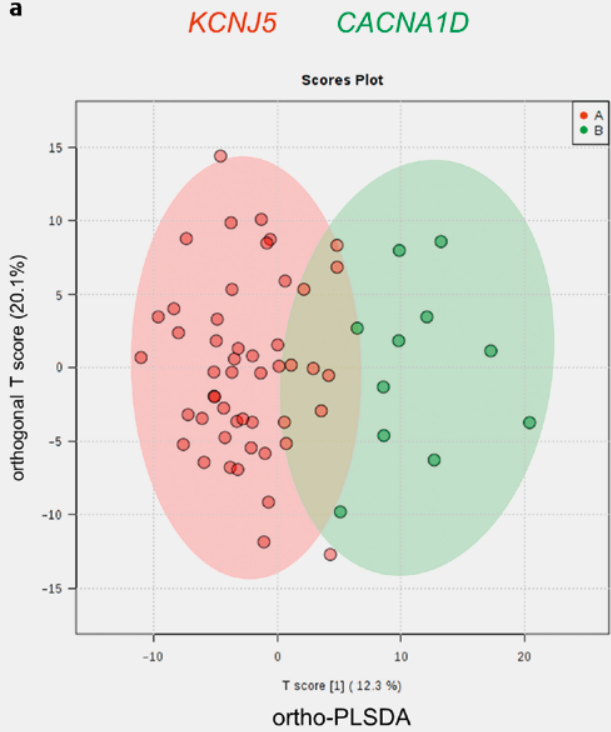

b
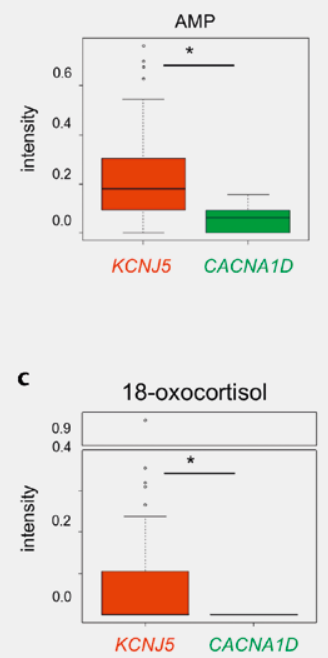

purine metabolism
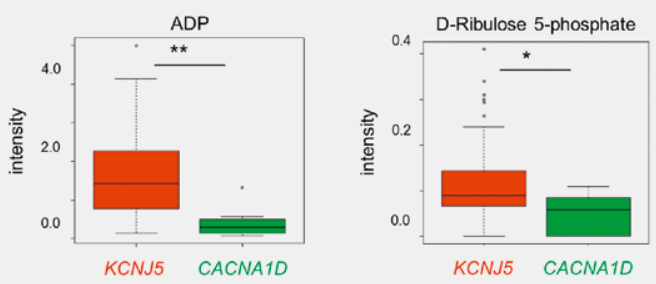

-Fig. 4 a The results of orthogonal partial least squares discriminant analysis (ortho-PLSDA), which identified separate clusters for KCNJ5- and CACNA1D-mutated aldosterone-producing adenomas (APAs) based on metabolome profiles. $\mathbf{b}$ Significant difference in metabolites related to purine metabolism were found between KCNJ5- and CACNA1D-mutated APAs (adjusted $p=0.010,0.003$, and 0.047 , respectively). $c$ Significant changes in the intensities of 18-oxocoritsol $(p=0.020)$ and 18-hydroxycortisol $(p<0.001)$ were identified between KCNJ5- and CACNA1D-mutated APAs. Mann-Whitney U-tests were used for statistical analysis. * $\mathrm{p}<0.05 ;{ }^{*}{ }^{*} \mathrm{p}<0.01$. (Reprinted from [48] with permission from American Society for Clinical Investigation) [rerif].

creased the molecular specificity and sensitivity in the rabbit adrenal gland using sodium-doped gold-assisted LDI-enhanced MSI (high lateral resolution of $25 \mu \mathrm{m}$ ) to show distributions of cholesterol, different cholesterol esters (CE) and triacylglycerols (TAGs) [58].

Normal human adrenal gland molecular imaging has been performed by Sun et al. using a comprehensive MADLI-FTICR-MSI [20]. As mentioned before, a broad spectrum of metabolites and hormones, including epinephrine, norepinephrine, dehydroepiandrosterone sulfate, pregnenolone sulfate, cholesterol sulfate, AMP, ADP, ATP, UDP-glucose, UDP- $N$-acetylglucosamine, glucose monophosphate, citrate, palmitic acid, adrenic acid, and phosphatidylinositol, can be measured via this method. Functional adrenal zonation assessed by IHC demonstrates an exact co-localization of sulfated steroid hormone with zone specific IHC markers in the human adrenal gland. A clear separation of the cortex and medulla was observed at the level of individual metabolites by heat map-based clustering analysis of discriminative metabolites. At the comprehensive metabolic pathway level, pathway enrichment analysis revealed enhanced steroid hormone and fatty acid synthesis in the adrenal cortex, which is in line with the known steroid hormone production in the cortex. Elevated activity of the pentose phosphate pathway and glycolysis and nucleotide metabolism in the medulla was found, reflecting the high energy consumption in the medulla. A spatial segmentation map based on unsupervised hierarchical clustering generated not only a clear separation between the cortex and medulla ( $\triangleright$ Fig. 3 , level 1 ) but also a virtually molecular-based map that matched perfectly with the histological morphological features of adrenal gland, including the zona glomerulosa (ZG), zona fasciculata (ZF), zona reticularis (ZR), and medulla ( $\triangleright$ Fig. 3, level 2). Further improved spatial segmentation created
10 individual molecular-defined zones corresponding to functionally distinct parts that are not completely reflected by the histological layers of the adrenal gland ( $\vee$ Fig. 3, level 3 ) [20]. Among these identified zones, molecular zones 1-6 represented the cortex area and molecular zones 7-10 corresponded to the medulla area. The relevance of molecular-based zonation, although not well-elucidated, is likely to reflect functional adaptation to the interplay between the adrenal medulla and cortex.

\section{Molecular Imaging of whole-body animal tissue section (FF tissue)}

MSI has also been used for whole-body sections from different model animals such as rats, mice $[59,60]$. Molecular structures enriched in the adrenal gland could be accessible via whole-body molecular imaging. As an example, MALDI-MSI of mice has identified one phospholipid ( $\mathrm{m} / \mathrm{z}$ 810.60) enriched in the adrenal gland [61]. Whole-body MSI simultaneously detects the presence of molecules in different organs, thus providing a way for studying the relation of adrenal gland with other organs. Another study observed the presence of the antitumor candidate drug $(S)-(+)$-deoxytylophorinidine (CAT) after drug dosing in a whole-body molecular image via air flow-assisted ionization in the DESI mode (AFADESI)imaging mass spectrometry (IMS) [62]. High drug concentration in the adrenal gland was found 20 min after dosing and was steadily conserved 2 hours after dosing. The visualization of exogenous drug disposition in multiple organs in the whole-body section through MSI allows the direct discovery of the organ targeted by drug candidate. The prediction of pharmacological activity and potential toxicity could be one potential application of such method. 

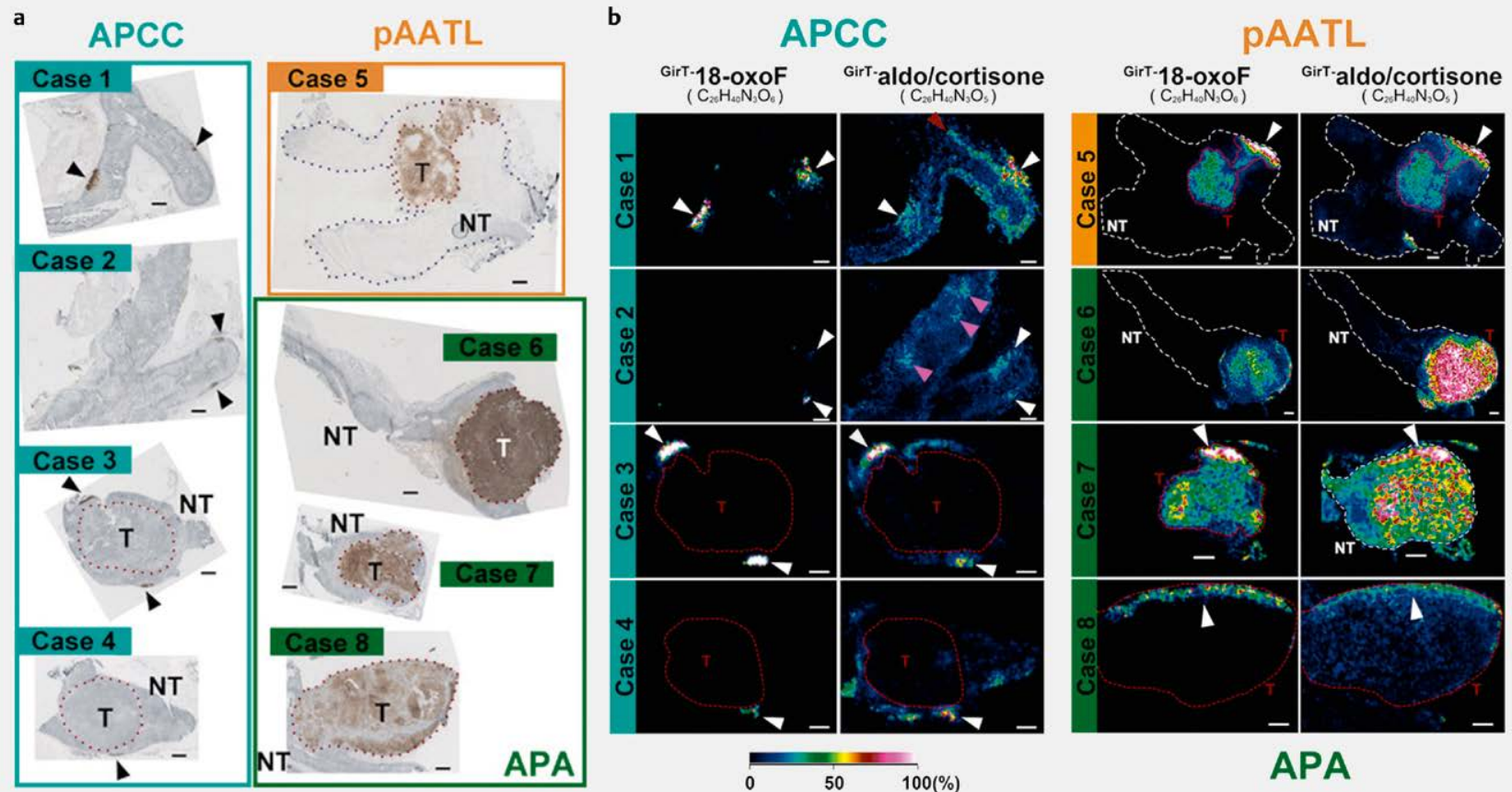

- Fig. 5 a Immunohistochemistry (IHC) for CYP11B2 in human adrenalectomized samples. Cases 1-4 (blue background), case 5 (orange background) and cases 6-8 (green background) show IHC results for aldosterone-producing cell clusters (APCCs; arrowheads), possible APCC-to-aldosterone-producing adenoma (APA) transitional lesions (PAATLs), and APAs, respectively. In cases 3-8; T: Tumor area within red dotted lines, NT: Non-tumor area outside of the red dotted line. Bars represent $1 \mathrm{~mm}$. b Matrix-assisted laser desorption/ionization (MALDI) imaging of APCCs (cases 1-4, blue background), pAATLs (case 5, orange background), and APAs (cases 5-8, green background) using Fourier-transform ion cyclotron resonance mass spectrometry (FT-ICR-MS). GirT-18-oxoF shows the distribution of derivatized 18-oxocortisol. GirT-aldo/cortisone demonstrates the distribution of derivatized aldosterone and cortisone. In cases $1-4$, white arrowheads correspond to black arrowheads indicating APCCs in $>$ Fig. $\mathbf{5 a}$. In cases 3-8, T (tumor region within orange dotted lines) and NT (non-tumor region outside of the orange dotted line) correspond to CYP11B2 staining in > Fig. 5a. Bars represent $1 \mathrm{~mm}$. (Reprinted from [49] with permission from Wolters Kluwer Health, Inc.) [rerif].

\section{Clinicopathologic application of MSI in the study of adrenal tumors}

The dysfunction of the adrenal gland could present as an excess or insufficiency of adrenal hormones in conjunction with relevant symptoms. For example, overproduction of glucocorticoids causes Cushing's syndrome, whereas excessive aldosterone results in hyperaldosteronism [63-65]. The most common benign adrenal tumor is adrenocortical adenoma, most of which are aldosterone-producing or cortisol-producing adrenocortical adenoma. Adrenocortical carcinoma (ACC) is a rare endocrine tumor with great clinical significance due to its high malignancy potential [66]. Pheochromocytomas are tumors of chromaffin cells, which most commonly arise in the adrenal medulla and are usually not metastatic $[67,68]$. In all cases, adrenal diseases and tumors have the potential to impair quality of life and may be life-threatening.

\section{In situ metabolomics of aldosterone-producing adenomas (APAs) by MSI and its clinical implications (FFPE tissue)}

MALDI-FTICR-MSI was applied to a cohort of 136 FFPE tissue cores of patients with a unilateral primary aldosteronism, which allowed for a comprehensive metabolite profile analysis and association with genotype/phenotype, IHC of steroidogenic enzymes and clinical features/outcomes [48]. This study identified 137 significantly different metabolites between KCNJ5- and CACNA1D-mutated APAs, including metabolites associated with steroidogenesis and purine metabolism. An increase in purine synthesis in APAs with KCNJ5 mutations could reflect the promotion of cell proliferation, which is also connected to the larger tumor size observed in KCNJ5-mutated APAs (> Fig. 4). High levels of 18-oxocortisol and 18-hydroxylcortisol were found in KCNJ5-mutated APAs ( $>$ Fig. 4). Accordingly, high plasma levels of 18-oxocortisol and 18-hydroxylcortisol were identified in patients with KCNJ5-mutated APA and were shown to be predictive of KCNJ5 mutation. Distinct metabolome profiles between these two gene mutations were further supported by orthogonal partial least squares discriminant analysis (ortho-PLSDA) clustering ( $\triangleright$ Fig. 4). Regarding steroids and steroidogenesis enzymes, 18-oxocortisol and 18-hydroxylcortisol were inversely correlated with CYP11B1 but not CYP11B2 in APA tissues, which might be explained by an overall high expression of CYP11B2above a threshold critical for detecting 18 steroid level differences. CYP11B2 has been shown to convert 11-deoxycortisol to 18-oxocortisol and 18-hydroxylcortisol, whereas CYP11B1 only produces 18-hydroxylcortisol [69]. Moreover, both 18-oxocortisol intensity and CYP11B1 expression levels in tumor tissue are predictive of the clinical outcome, independent of other clinical parameters [48]. For example, complete clinical success after surgery was linked to high intensity of 18-oxocortisol or low staining of 
a

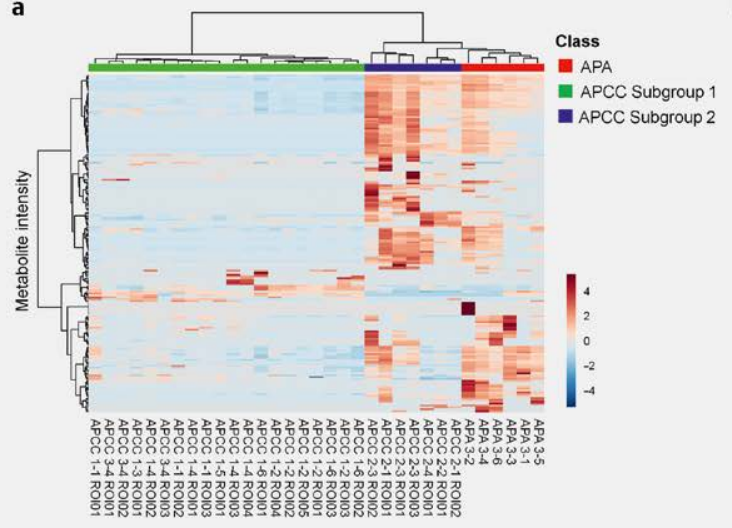

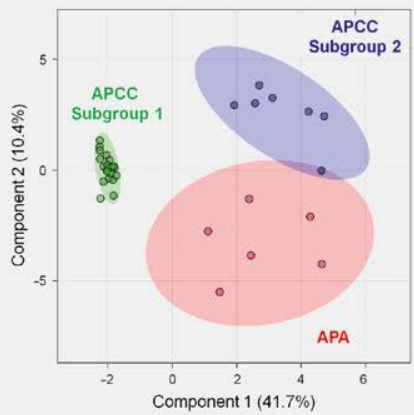

c

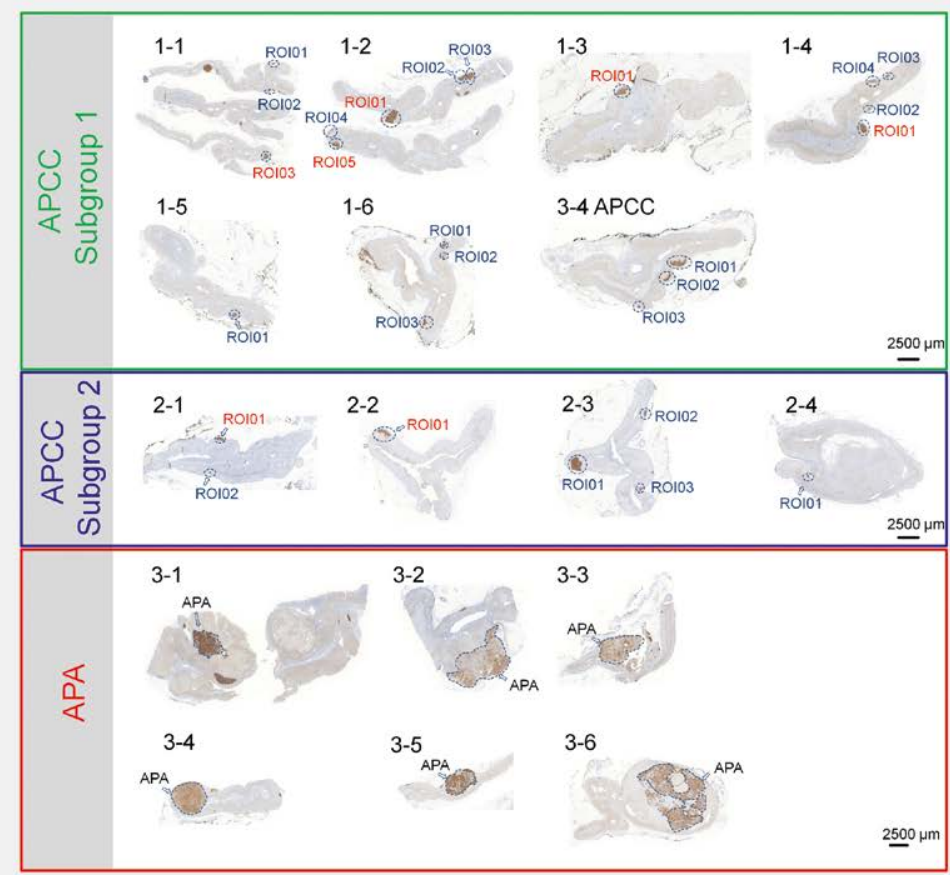

- Fig. 6 Hierarchical clustering and component analysis demonstrated different metabolic profiles in APCC subgroup 1, APCC subgroup 2 and APAs. a Peak lists with respective intensities were uploaded to MetaboAnalyst. Each colored cell corresponds to an intensity value, with samples in rows and features in columns. Euclidean distance and Ward's method were applied for cluster analysis. b Component analysis using sPLSDA identified 3 patterns of metabolites comprising 2 subgroups of clearly separated APCCs (subgroup 1 and subgroup 2) and the APA group. c CYP11B2 (aldosterone synthase) immunohistochemistry of adrenal samples included in the metabolic analyses showing APCCs in subgroup 1, APCCs in subgroup 2 and APAs. ROI (region of interest) identification numbers are shown for each APCC analyzed. (Reprinted from [72] with permission from Wolters Kluwer Health, Inc.) [rerif].

CYP11B1, demonstrating the importance of CYP11B1 over CYP11 $\mathrm{B} 2$ in tumor tissue with regard to the clinical outcome. This association between 18 steroid levels, CYP11B1 and clinical outcome also may be related to the glucocorticoid co-secretion observed in APAs, and also may be related to "Connshing" syndrome [70, 71].

\section{MSI imaging in the adrenal cortex suggests patho- physiological progression from aldosterone- producing cell clusters (APCCs) to APA (FF and FFPE tissue)}

APCC s can be identified by IHC staining of strong aldosterone synthase (CYP11B2) expression without expression of steroid 11ß-hy- droxylase (cortisol-synthesizing enzyme, CYP11B1), even in the non-primary aldosteronism (AP) producing adult cortex ( $>$ Fig. 5a, cases 1-4). Thus, APCCs seem to produce aldosterone autonomously. The accumulation of aldosterone and 18-oxocortisol in CYP11B2-expressive lesions (APCC) was confirmed by Sugiyama et al. by visualizing GirT derivatized aldosterone on FF adrenal samples [49]. The distribution patterns of 18-oxocortisol and aldosterone in APCC and APA tissues ( $\vee$ Fig. $\mathbf{5 b}$ ) have led to a progressive development hypothesis of APA generation in which APCCs develop into APAs via APCC-to-APA transitional lesions (pAATLs: • Fig. $\mathbf{5 b}$, case 5). These PAATLs can be divided into a sub-capsular APCClike region and an inner APA-like region. The APCC-like region of a 
a

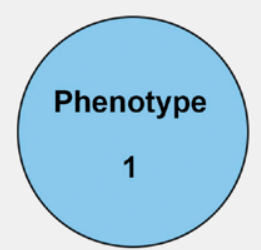

Estradiol-17ß 3-sulfate Low abundance Poor prognosis $n=5$
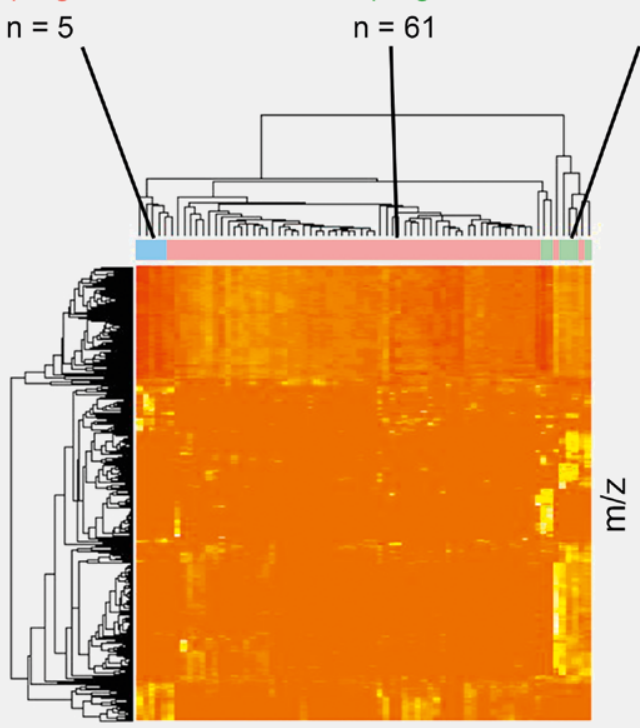

patients

b

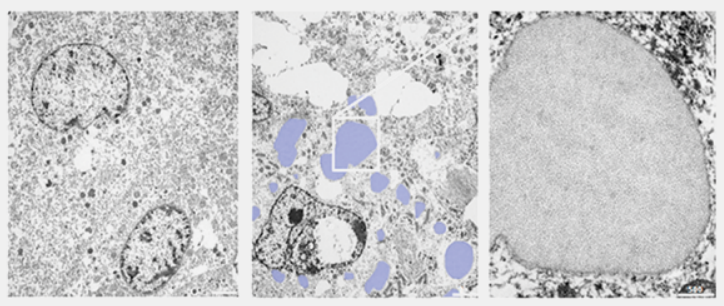

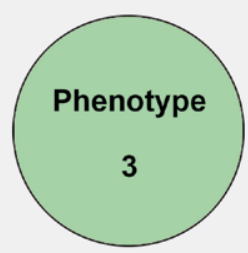

Estradiol-17 $\beta$ 3,17-disulfate Molecule is present Poor prognosis $\mathrm{n}=6$

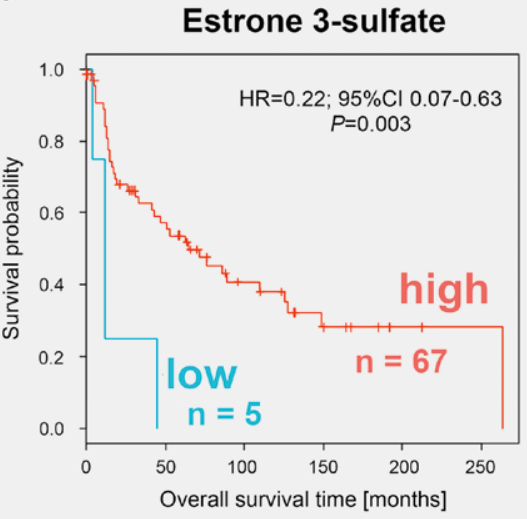

d

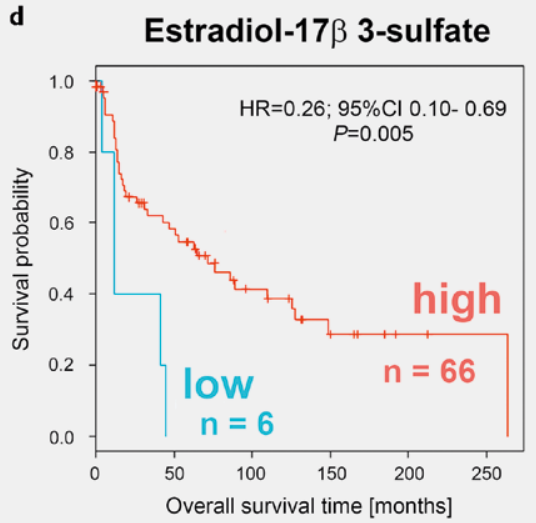

e Estradiol-17 $\beta$ 3,17-disulfate

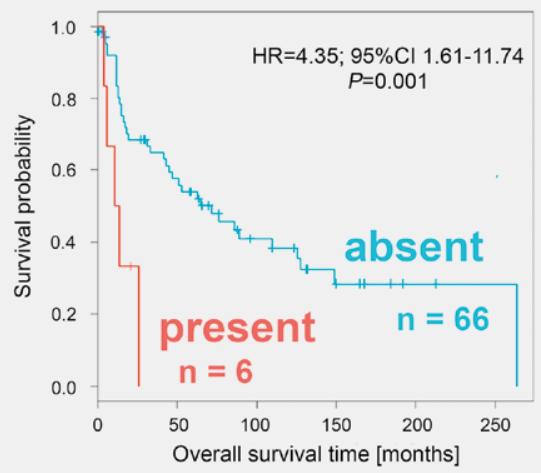

- Fig. 7 a Hierarchical cluster analysis of molecular $\mathrm{m} / \mathrm{z}$ separates adrenocortical carcinoma (ACC) patient cohorts into three phenotypes, namely phenotype 1, phenotype 2, and phenotype 3. b Electron microscopy of phenotype 1 tissue (left panel) and phenotype 3 tissue (center panel), and vacuoles in phenotype 3 (highlighted in blue in the center panel) are enlarged as regions of interest (ROI, right panel). c,d,e Survival probabilities based on Kaplan-Meier analyses for patients with high (red) and low (blue) estrone-3-sulfate (E1S) and estradiol-17 $\beta$-3-sulfate (E2S) levels, and patients with (red) or without (blue) disulfated steroid estradiol-17ß-3,17-disulfate (E2S2). (Reprinted from [21] by permission of Oxford University Press) [rerif].

pAATL consists of aldosterone-producing cells like an APCC, whereas the APA-like region of a PAATL contains both aldosterone- and cortisol-producing cells like a small size APA. In one case with a large CYP11B2-positive APA ( Fig. 5b, case 8), both an aldosterone-producing area and a nonfunctional area without aldosterone were identified via imaging; the latter nonfunctional area may have been due to a lack of precursor steroids, including progesterone. Both plasma 18-oxocortisol and aldosterone levels are increased in pa- tients with APAs. This study found accumulation of 18-oxocortisol in the CYP11B2-positive APCC regions, as well as in the APA regions, with either heterogeneous or relatively homogenous distributions ( Fig. 5b) [49]. By comparing the steroid distributions imaged via MSI analysis ( $\mathbf{F i g}$. $\mathbf{5 b}$ ) with the distributions of steroidogenesis enzymes identified via IHC staining ( $>$ Fig. $5 a$ ), this study generated the hypothesis that 18-oxocortisol in the APCC region results from 18-oxidization of cortisol by CYP11B2, and that the cortisol 


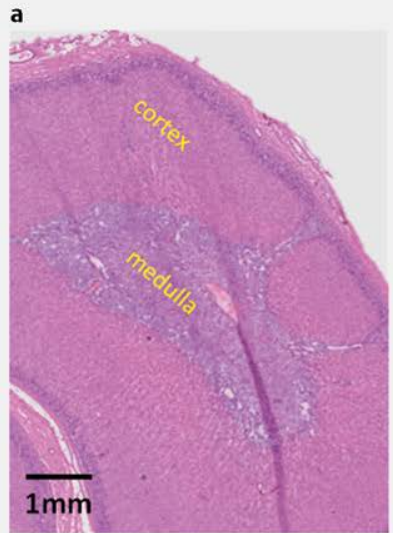

H\&E Stained Porcine Adrenal Gland

e

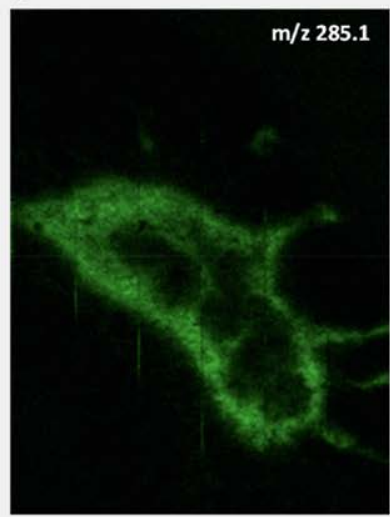

Epinephrine + 4-(N-Me)Py ${ }^{+}(\mathrm{OH})_{2}$ b

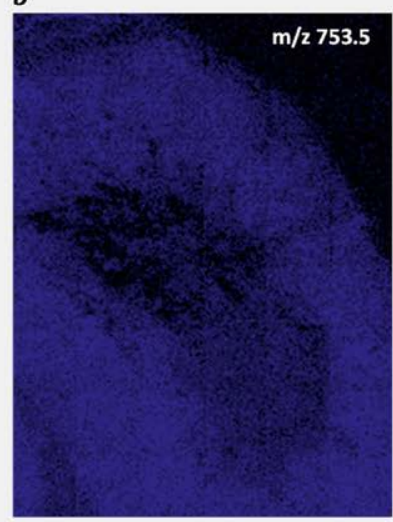

$\mathrm{m} / \mathrm{z} 753.5$

f

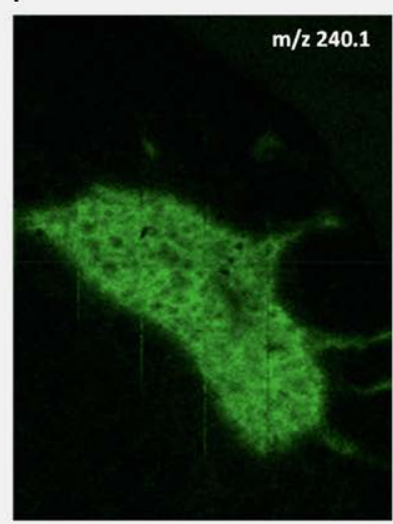

Fragment Ion of

Epinephrine/Norepinephrine + 4-(N-Me)Py ${ }^{+} \mathrm{B}(\mathrm{OH})_{2}$

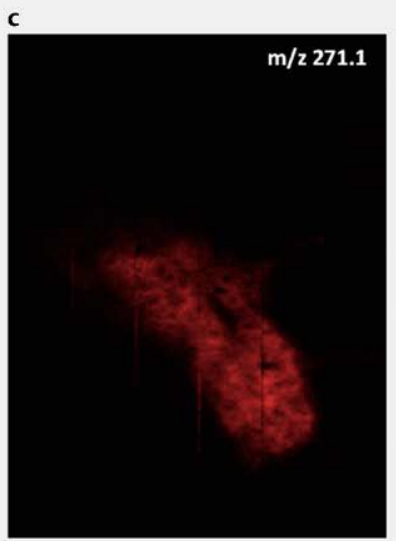

Norepinephrine+4-(N-Me)Py ${ }^{+} \mathrm{B}(\mathrm{OH})_{2}$

g

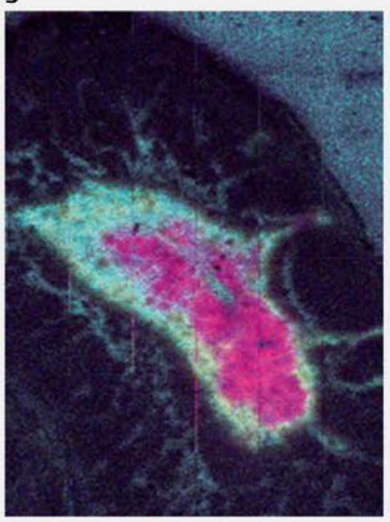

Merged Ion Images $\mathrm{m} / \mathrm{z} 255.1+271.1+285.1$ d

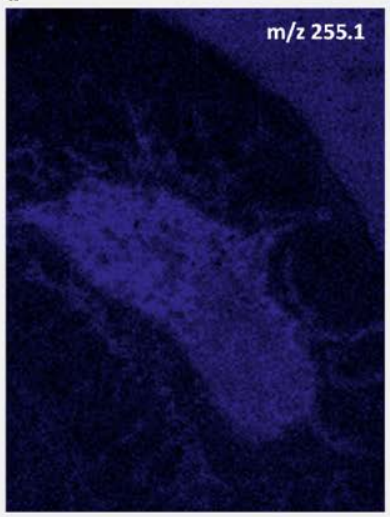

Dopamine + 4-(N-Me)Py+B(OH $)_{2}$

h

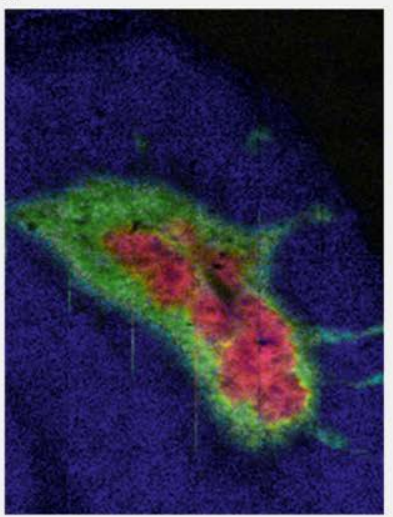

Merged Ion Images $\mathrm{m} / \mathrm{z} 753.5+271.1+285.1$

Fig. 8 The distribution of 4-( $\mathrm{N}$-methyl)pyridinium boronic acid $\left[4-(\mathrm{N}-\mathrm{Me}) \mathrm{PyB}(\mathrm{OH})_{2}\right]$ derivatized catecholamines in the porcine adrenal gland was analyzed by GCIB-ToF-SIMS (30 $\mu \mathrm{m}$ per pixel). a Hematoxylin and eosin ( $\mathrm{H} \& \mathrm{E})$ staining of adrenal cortex and medulla. $\mathbf{b}$ An unidentified peak with $\mathrm{m} / \mathrm{z}$ 753.5. c Norepinephrine-4-(N-Me)PyB(OH$)_{2}$ with m/z 271.1. d Dopamine-4-(N-Me)PyB(OH $)_{2}$ with m/z 255.1. e Epinephrine-4-(N-Me)PyB(OH) 2 with $\mathrm{m} / \mathrm{z}$ 285.1. f fragment ion of epinephrine/norepinephrine-4-(N-Me)PyB(OH$)_{2} . \mathbf{g}$ and $\mathbf{h}$ Merged ion images of norepinephrine-4-( $\left.\mathrm{N}-\mathrm{Me}\right) \mathrm{PyB}(\mathrm{OH})_{2}$ (red), epinephrine-4-(N-Me)PyB $(\mathrm{OH})_{2}$ (green), dopamine-4-(N-Me)PyB(OH)$)_{2}$ (blue, g) or unidentified peak with $\mathrm{m} / \mathrm{z} 753.5$ (blue, h). (Reprinted from [51] with permission from American Chemical Society) [rerif].

diffused to the APCC region from the surrounding ZF. The hypothesis that cortisol moves from CYPB11-active region to APCCs and that cortisol is further oxidized by CYP11B2 into 18-oxocortisonl and 18-hydroxycortisol were further supported by visualizing cortisol in the human adrenal gland as mentioned before [52]. Cortisol were found to be absent in all APCCs where aldosterone was accumulated.

The most recent publication of Sun et al. used used in situ metabolic imaging analysis by MALDI-FT-ICR-MSI of FFPE adrenals resected from patients with unilateral primary aldosteronism to investigate the potential existence of diverse metabolic phenotypes of APCCs [72]. The analysis covered a wide range of central metabolic and steroid hormone biosynthesis pathways and determined heterogeneous patterns of metabolites associated with APCCs.

Specific distribution patterns of metabolites were associated with APCCs and classified 2 separate APCC subgroups (subgroups 1 and 2) indistinguishable by CYP11B2 immunohistochemistry.
Metabolic profiles of APCCs in subgroup 1 were tightly clustered and distinct from subgroup 2 and APAs. Multiple APCCs from the same adrenal displayed metabolic profiles of the same subgroup ( $\triangleright$ Fig. 6). Metabolites of APCC subgroup 2 were highly similar to the APA group and indicated enhanced metabolic pathways favoring cell proliferation compared with APCC subgroup 1. In conclusion, the results demonstrate specific subgroups of APCCs with strikingly divergent distribution patterns of metabolites. One subgroup displays a metabolic phenotype convergent with APAs and may represent the progression of APCCs to APAs.

\section{Prognostic relevance of steroid sulfation detected by MSI in adrenocortical carcinoma (ACC) (FFPE tissue)}

Molecules measured by MSI could be used in conjunction with genomic and RNA expression data to determine the molecular phenotype of tumors. As shown in > Fig. 7a, MALDI-FTICR-MSI analysis identified the molecular phenotype of 72 ACCs based on steroid 
hormone metabolites [21]. In this study, high levels of estradiol sulfate (E2S) and estrone-3-sulfate (E1S) were significantly associated with a good ACC prognosis via both univariate Kaplan-Meier analysis and after multivariate adjustment for tumor stage, age, and sex ( $\mathbf{F i g}$. 7c and d). This finding supports the idea that active steroid sulfation is a marker of a less aggressive ACC phenotype. Moreover, the expression of sulfotransferase SULT2A1 in the same tissue samples had similar prognostic power. This finding was validated in two published data sets, and suggests that SULT2A1 is the enzyme responsible for the synthesis of E1S and E2S. Beyond the known steroid sulfates, disulfated steroid estradiol-17 $\beta$-3,17-disulfate (E2S2) also was found in six samples, first by searching for the calculated theoretical mass in the measured spectrum and then validating by MS/MS. Its presence in these six patients was associated with a particularly poor overall survival ( $\triangleright$ Fig. 7 e). Multiple membrane-delimited vacuoles were found only in these tumors with E2S2, supporting the intracellular accumulation of E2S2 ( $\triangleright$ Fig. 7b). This study thus highlights the potential of steroid hormone sulfation to predict a patient's prognosis and to stratify patients by treatment.

\section{MSI of catecholamines in the adrenal medulla (FF tissue)}

Although catecholamines in the medulla region are accessible via different MSI techniques such as DESI-MSI or high-resolution MALDIFTICR-MSI, as previously mentioned [20,56], a few studies have also applied derivatization for catecholamines visualization. Manier et al. [50] improved the sensitivity of the medulla origin hormones dopamine, epinephrine and norepinephrine by pre-coating the target with 4-hydroxy-3-methoxycinnamaldehyde (CA) as a derivatization reagent in a MALDI ion trap MSI analysis. The signals for these three target compounds from underivatized analyses were very low with a non-specific distribution in their analysis, indicating insufficient sensitivity for distinguishing target compounds from the chemical background or matrix interference. In contrast, derivatization followed by MALDI-MSI with MSn analysis resulted in substantially improved sensitivity, allowing a clear visualization of the respective hormones in situ in the medulla region. Particularly, $\mathrm{MS}^{3}$ was necessary to produce the specific fragments to enable visualization of derivatized norepinephrine. A more accurate localization of dopamine, epinephrine and norepinephrine in the modular region also was achieved in another study [51]. By applying 4-( $\mathrm{N}$-methyl)pyridiniumboronic acid [4(NMe)PyB $(\mathrm{OH})_{2}$ ] derivatization, both LDI-TOF-MSI and gas cluster ion beam (GCIB)-TOF-SIMS analysis yielded a similar characteristic spatial distribution of epinephrine, norepinephrine and dopamine in porcine adrenal gland tissue sections; these compounds localize in the peripheral, central and entire medulla region, respectively, as shown in $>$ Fig. 8, [51]. In another study, the derivatization reagent $p$ - $N, N, N$-trimethylammonioanilyl $N$ '-hydroxysuccinimidyl carbamate iodide (TAHS) was applied to successfully discriminate enriched catecholamine species in the pheochromocytoma region [52].

\section{MSI for utilizing the pheochromocytoma (PC12) cell model (FF tissue)}

A few studies have applied MSI techniques, mainly secondary ion (SI) MSI, for measuring the pheochromocytoma (PC12) cell model, which is widely used as a model system for studying exocytosis [73]. PC12 cells release catecholamines by exocytosis and differentiate into neuron-like cells upon treatment with nerve growth factor [74]. TOFSIMS was used to image lipids fragments in freeze fractured rat pheochromocytoma (PC12) cells on a single-cell level [73-75]. Dopamine localization was identified in the vesicular compartments of pheochromocytoma (PC12) cells by correlating TEM and NanoSIMS images of single vesicles [76]. In a drug-related study, a decrease of lipids in PC12 cells treated with cisplatin was observed, suggesting that cisplatin affects exocytotic release by alternating cell membrane lipids [77].

\section{Conclusion and Perspectives}

The innovative and continuously developed MALDI-MSI technology has demonstrated great power for both basic study and clinical/ pre-clinical study of the adrenal gland under both physiological and pathophysiological conditions. Its application has enabled a deeper understanding of the molecular adaptation underlying adrenal diseases, particularly adrenal tumors. Retrospective studies of patient cohorts allows for the investigation of tumorigenesis and clinically relevant abnormal steroidogenesis at the molecular level, adding one more level of information to existing enzyme and genetic data. Clinically relevant patient stratification of adrenal tumors via molecular identification may pave the way for novel and robust tissue biomarker based precision medicine and therapeutic decision making. Future studies may extend currently existing clinicopathologic application of simultaneous imaging of hormones, metabolites, drugs and their metabolites in the adrenal gland and other endocrine organs from patients and animal models. In the future, prospective cohort studies can also validate steroid fingerprinting as markers for malignancy and the staging of newly detected adrenal neoplasms. In addition to diagnostics, steroid metabolism may serve as an objective and refined method for monitoring the treatment of adrenal patients. Finally, the extension of this application to not only adrenal disease but also other systemic diseases involving adrenal dysfunction has promising possibilities.

\section{Funding Information}

Deutsche Forschungsgemeinschaft (CRC/TRR 205 "The Adrenal: Central Relay in Health and Disease", projects S01 to A.W.). Deutsche Krebshilfe (no. 70112617 to A.W.)

\section{Conflicts of Interest}

The authors declare that they have no conflict of interest.

\section{References}

[1] Dilillo M, Heijs B, McDonnell LA. Mass spectrometry imaging: How will it affect clinical research in the future? Expert Rev Proteomics 2018; 15: 709-716

[2] Buck A, Aichler M, Huber $K$ et al. In situ metabolomics in cancer by mass spectrometry imaging. Adv Cancer Res 2017; 134: 117-132 
[3] Qi M, Philip MC, Yang N et al. Single cell neurometabolomics. Acs Chem Neurosci 2018; 9: 40-50

[4] Arentz G, Mittal P, Zhang C et al. Applications of mass spectrometry imaging to cancer. Adv Cancer Res 2017; 134: 27-66

[5] Smith A, Piga I, Galli M et al. Matrix-assisted laser desorption/ ionisation mass spectrometry imaging in the study of gastric cancer: A mini review. Int J Mol Sci 2017; 18: pii E2588. doi: 10.3390/ ijms18122588

[6] Phillips L, Gill AJ, Baxter RC. Novel prognostic markers in triple-negative breast cancer discovered by MALDI-mass spectrometry imaging. Front Oncol 2019; 9: 379

[7] Peng X, Chen Z, Farshidfar F et al. Molecular Characterization and clinical relevance of metabolic expression subtypes in human cancers. Cell Rep 2018; 23: 255-269.e254

[8] Hanahan D, Weinberg RA. Hallmarks of cancer: The next generation. Cell 2011; 144: 646-674

[9] Papathomas TG, Sun N, Chortis V et al. Novel methods in adrenal research: A metabolomics approach. Histochem Cell Biol 2019; 151: 201-216

[10] Patel E. Fresh frozen versus formalin-fixed paraffin embedded for mass spectrometry imaging. Methods Mol Biol 2017; 1618: 7-14

[11] Buck A, Ly A, Balluff B et al. High-resolution MALDI-FT-ICR MS imaging for the analysis of metabolites from formalin-fixed, paraffin-embedded clinical tissue samples. J Pathol 2015; 237: 123-132

[12] Ly A, Buck A, Balluff B et al. High-mass-resolution MALDI mass spectrometry imaging of metabolites from formalin-fixed paraffin-embedded tissue. Nat Protoc 2016; 11: 1428-1443

[13] Buck A, Heijs B, Beine B et al. Round robin study of formalin-fixed paraffin-embedded tissues in mass spectrometry imaging. Anal Bioanal Chem 2018; 410: 5969-5980

[14] Haase M, Willenberg HS, Bornstein SR. Update on the corticomedullary interaction in the adrenal gland. Endocr Dev 2011; 20: 28-37

[15] Hundemer GL, Vaidya A. Primary aldosteronism diagnosis and management: A clinical approach. Endocrinol Metab Clin North Am 2019; 48: 681-700

[16] van der Heijden C, Keating ST, Groh L et al. Aldosterone induces trained immunity: The role of fatty acid synthesis. Cardiovasc Res 2020; 116: 317-328

[17] Liu B, Zhang TN, Knight JK et al. The glucocorticoid receptor in cardiovascular health and disease. Cells 2019; 8: pii E1227. doi: $10.3390 /$ cells 8101227

[18] Russell G, Lightman S. The human stress response. Nat Rev Endocrinol 2019; 15: 525-534

[19] Dekker T], Jones EA, Corver WE et al. Towards imaging metabolic pathways in tissues. Anal Bioanal Chem 2015; 407: 2167-2176

[20] Sun N, Wu Y, Nanba K et al. High-resolution tissue mass spectrometry imaging reveals a refined functional anatomy of the human adult adrenal gland. Endocrinology 2018; 159: 1511-1524

[21] Sun N, Kunzke T, Sbiera S et al. Prognostic relevance of steroid sulfation in adrenocortical carcinoma revealed by molecular phenotyping using high-resolution mass spectrometry imaging. Clin Chem 2019; 65: 1276-1286

[22] Heeren RMA. Getting the picture: The coming of age of imaging MS. International J Mass S pectrom 2015; 377: 672-680

[23] Vaysse PM, Heeren RMA, Porta T et al. Mass spectrometry imaging for clinical research - latest developments, applications, and current limitations. Analyst 2017; 142: 2690-2712

[24] Caprioli RM, Farmer TB, Gile J. Molecular imaging of biological samples: localization of peptides and proteins using MALDI-TOF MS. Anal Chem 1997; 69: 4751-4760

[25] Chaurand P, Schwartz SA, Billheimer D et al. Integrating histology and imaging mass spectrometry. Anal Chem 2004; 76: 1145-1155
[26] Todd PJ, Schaaff TG, Chaurand P et al. Organic ion imaging of biological tissue with secondary ion mass spectrometry and matrix-assisted laser desorption/ionization. J Mass Spectrom 2001; 36: 355-369

[27] Abdelhamid HN. Organic matrices, ionic liquids, and organic matrices@nanoparticles assisted laser desorption/ionization mass spectrometry. Trends Anal Chem 2017; 89: 68-98

[28] Norris JL, Caprioli RM. Analysis of tissue specimens by matrix-assisted laser desorption/ionization imaging mass spectrometry in biological and clinical research. Chem Rev 2013; 113: 2309-2342

[29] Cobice DF, Goodwin RJ, Andren PE et al. Future technology insight: mass spectrometry imaging as a tool in drug research and development. Br J Pharmacol 2015; 172: 3266-3283

[30] Duenas ME, Larson EA, Lee Y]. Toward mass spectrometry imaging in the metabolomics scale: Increasing Metabolic Coverage Through Multiple On-Tissue Chemical Modifications. Front Plant Sci 2019; 10: 860

[31] Balluff B, Schone C, Hofler H et al. MALDI imaging mass spectrometry for direct tissue analysis: technological advancements and recent applications. Histochem Cell Biol 2011; 136: 227-244

[32] McDonnell LA, van Remoortere A, de Velde $\mathrm{N}$ et al. Imaging mass spectrometry data reduction: Automated feature identification and extraction. J Am Soc Mass Spectrom 2010; 21: 1969-1978

[33] Wishart DS, Feunang YD, Marcu A et al. HMDB 4.0: The human metabolome database for 2018. Nucleic Acids Res. 2018; 46: D608-D617

[34] Palmer A, Phapale P, Chernyavsky I et al. FDR-controlled metabolite annotation for high-resolution imaging mass spectrometry. Nat Meth 2017; 14: 57-60

[35] Vinaixa M, Schymanski EL, Neumann S et al. Mass spectral databases for LC/MS- and GC/MS-based metabolomics: State of the field and future prospects. Trend Anal Chem 2016; 78: 23-35

[36] Ceglarek U, Leichtle A, Brugel $M$ et al. Challenges and developments in tandem mass spectrometry based clinical metabolomics. Mol Cell Endocrinol 2009; 301: 266-271

[37] Bowen BP, Northen TR. Dealing with the unknown: Metabolomics and metabolite atlases. J Am Soc Mass Spectrom 2010; 21: 1471-1476

[38] Alexandrov T. MALDI imaging mass spectrometry: Statistical data analysis and current computational challenges. BMC Bioinformatics 2012; 13 (Suppl 16) : S11

[39] Galli M, Zoppis I, Smith A et al. Machine learning approaches in MALDI-MSI: Clinical applications. Expert Rev Proteomics 2016; 13 685-696

[40] Balluff B, Buck A, Martin-Lorenzo M et al. Integrative clustering in mass spectrometry imaging for enhanced patient stratification. Proteomics Clin Appl 2019; 13: e1800137

[41] Leuschner ], Schmidt M, Fernsel P et al. Supervised non-negative matrix factorization methods for MALDI imaging applications. Bioinformatics 2019; 35: 1940-1947

[42] Abdelmoula WM, Balluff B, Englert $S$ et al. Data-driven identification of prognostic tumor subpopulations using spatially mapped t-SNE of mass spectrometry imaging data. Proc Natl Acad Sci USA 2016; 113 : 12244-12249

[43] Balluff B, Rauser S, Ebert MP et al. Direct molecular tissue analysis by MALDI imaging mass spectrometry in the field of gastrointestinal disease. Gastroenterology 2012; 143: 544-549.e542

[44] Kurreck A, Vandergrift LA, Fuss TL et al. Prostate cancer diagnosis and characterization with mass spectrometry imaging. Prostate Cancer Prostatic Dis 2018; 21: 297-305

[45] Quanico J, Franck J, Wisztorski M et al. Progress and potential of imaging mass spectrometry applied to biomarker discovery. Methods Mol Biol 2017; 1598: 21-43 
[46] McDonnell LA, Angel PM, Lou S et al. Mass spectrometry imaging in cancer research: Future perspectives. Adv Cancer Res 2017; 134: 283-290

[47] Balluff B, Hanselmann M, Heeren RM. Mass spectrometry imaging for the investigation of intratumor heterogeneity. Adv Cancer Res 2017; 134: $201-230$

[48] Murakami M, Rhayem Y, Kunzke T et al. In situ metabolomics of aldosterone-producing adenomas. JCl Insight 2019; 4: pii 130356. doi: 10.1172 jci.insight.130356

[49] Sugiura Y, Takeo E, Shimma S et al. Aldosterone and 18-oxocortisol coaccumulation in aldosterone-producing lesions. Hypertension 2018; 72: $1345-1354$

[50] Manier ML, Spraggins JM, Reyzer ML et al. A derivatization and validation strategy for determining the spatial localization of endogenous amine metabolites in tissues using MALDI imaging mass spectrometry. J Mass Spectrom 2014; 49: 665-673

[51] Kaya I, Brulls SM, Dunevall J et al. On-Tissue Chemical Derivatization of Catecholamines Using 4-( N-Methyl)pyridinium Boronic Acid for ToF-SIMS and LDI-ToF Mass Spectrometry Imaging. Anal Chem 2018; 90: $13580-13590$

[52] Takeo E, Sugiura Y, Uemura T et al. Tandem mass spectrometry imaging reveals distinct accumulation patterns of steroid structural isomers in human adrenal glands. Anal Chem 2019; 91: 8918-8925

[53] Cobice DF, Mackay CL, Goodwin RJ et al. Mass spectrometry imaging for dissecting steroid intracrinology within target tissues. Anal Chem 2013; 85: 11576-11584

[54] Zhang Z, Sugiura Y, Mune T et al. Immunohistochemistry for aldosterone synthase CYP11B2 and matrix-assisted laser desorption ionization imaging mass spectrometry for in-situ aldosterone detection. Curr Opin Nephrol Hypertens 2019; 28: 105-112

[55] Malmberg P, Jennische E, Nilsson D et al. High-resolution, imaging TOF-SIMS: novel applications in medical research. Anal Bioanal Chem 2011; 399: 2711-2718

[56] Wu C, Ifa DR, Manicke NE et al. Molecular imaging of adrenal gland by desorption electrospray ionization mass spectrometry. Analyst 2010; 135: $28-32$

[57] Wang X, Han J, Pan J et al. Comprehensive imaging of porcine adrenal gland lipids by MALDI-FTMS using quercetin as a matrix. Anal Chem 2014; 86: 638-646

[58] Dufresne M, Masson JF, Chaurand P. Sodium-doped gold-assisted laser desorption ionization for enhanced imaging mass spectrometry of triacylglycerols from thin tissue sections. Anal Chem 2016; 88: 60186025

[59] Stoeckli M, Staab D, Schweitzer A et al. Imaging of a beta-peptide distribution in whole-body mice sections by MALDI mass spectrometry. J Am Soc Mass Spectrom 2007; 18: 1921-1924

[60] Khatib-Shahidi S, Andersson M, Herman JL et al. Direct molecular analysis of whole-body animal tissue sections by imaging MALDI mass spectrometry. Anal Chem 2006; 78: 6448-6456
[61] Chaurand P, Cornett DS, Angel PM et al. From whole-body sections down to cellular level, multiscale imaging of phospholipids by MALDI mass spectrometry. Mol Cell Proteomics 2011; 10: 0110.004259

[62] Luo Z, He J, Chen Y et al. Air flow-assisted ionization imaging mass spectrometry method for easy whole-body molecular imaging under ambient conditions. Anal Chem 2013; 85: 2977-2982

[63] Guignat L, Bertherat J. The diagnosis of Cushing's syndrome: An Endocrine Society Clinical Practice Guideline: Commentary from a European perspective. Eur J Endocrinol 2010; 163: 9-13

[64] Duan K, Mete O. Clinicopathologic correlates of primary aldosteronism. Arch Pathol Lab Med 2015; 139: 948-954

[65] Duan K, Gomez Hernandez K, Mete O. Clinicopathological correlates of adrenal Cushing's syndrome. J Clin Pathol 2015; 68: 175-186

[66] Erickson LA, Rivera M, Zhang J. Adrenocortical carcinoma: review and update. Adv Anat Pathol 2014; 21: 151-159

[67] Ctvrtlik F, Koranda P, Tichy T. Adrenal disease: A clinical update and overview of imaging. A review. Biomed Pap Med Fac Univ Palacky Olomouc Czech Repub 2014; 158: 23-34

[68] Jochmanova I, Pacak K. Pheochromocytoma: The first metabolic endocrine cancer. Clin Cancer Res 2016; 22: 5001-5011

[69] Lenders JWM, Williams TA, Reincke $M$ et al. Diagnosis of endocrine disease: 18-Oxocortisol and 18-hydroxycortisol: Is there clinical utility of these steroids? Eur J Endocrinol 2018; 178: R1-R9

[70] Arlt W, Lang K, Sitch AJ et al. Steroid metabolome analysis reveals prevalent glucocorticoid excess in primary aldosteronism. JCl Insight 2017; 2: pii 93136. doi: 10.1172/jci.insight.93136. eCollection 2017 Apr 20

[71] Gerards J, Heinrich DA, Adolf C et al. Impaired glucose metabolism in primary aldosteronism is associated with cortisol cosecretion. J Clin Endocrinol Metab 2019; 104: 3192-3202

[72] Sun N, Meyer LS, Feuchtinger A et al. Mass spectrometry imaging establishes 2 distinct metabolic phenotypes of aldosterone-producing cell clusters in primary aldosteronism. Hypertension 2020; 75: 634-644

[73] Lanekoff I, Kurczy ME, Hill R et al. Time of flight mass spectrometry imaging of samples fractured in situ with a spring-loaded trap system. Anal Chem 2010; 82: 6652-6659

[74] Roddy TP, Cannon DM Jr., Ostrowski SG et al. Identification of cellular sections with imaging mass spectrometry following freeze fracture. Anal Chem 2002; 74: 4020-4026

[75] Roddy TP, Cannon DM Jr., Meserole CA et al. Imaging of freeze-fractured cells with in situ fluorescence and time-of-flight secondary ion mass spectrometry. Anal Chem 2002; 74: 4011-4019

[76] Lovric J, Dunevall J, Larsson A et al. Nano secondary ion mass spectrometry imaging of dopamine distribution across nanometer vesicles. ACS Nano 2017; 11: 3446-3455

[77] Mohammadi AS, Li X, Ewing AG. Mass spectrometry imaging suggests that cisplatin affects exocytotic release by alteration of cell membrane lipids. Anal Chem 2018; 90: 8509-8516 